\title{
ON THE ROBUSTNESS OF THE FAT-TAILED DISTRIBUTION OF FIRM GROWTH RATES: A GLOBAL SENSITIVITY ANALYSIS
}

\author{
G. Dosi¹ ${ }^{* 1}$ M. C. Pereira ${ }^{\dagger 2}$ and M. E. Virgillito ${ }^{\ddagger 1}$ \\ ${ }^{1}$ Scuola Superiore Sant'Anna \\ ${ }^{2}$ University of Campinas
}

\begin{abstract}
Firms grow and decline by relatively lumpy jumps which cannot be accounted by the cumulation of small, "atom-less", independent shocks. Rather "big" episodes of expansion and contraction are relatively frequent. More technically, this is revealed by fat tail distributions of growth rates. This applies across different levels of sectoral disaggregation, across countries, over different historical periods for which there are available data. What determines such property? In Dosi et al., (2015) we implemented a simple multi-firm evolutionary simulation model, built upon the coupling of a replicator dynamic and an idiosyncratic learning process, which turns out to be able to robustly reproduce such a stylized fact. Here, we investigate, by means of a Kriging meta-model, how robust such "ubiquitousness" feature is with regard to a global exploration of the parameters space. The exercise confirms the high level of generality of the results in a statistically robust global sensitivity analysis framework.
\end{abstract}

\section{Keywords}

Firm Growth Rates, Fat Tail Distributions, Kriging Meta-Modeling, NearOrthogonal Latin Hypercubes, Variance-Based Sensitivity Analysis.

\section{JEL Classification}

C15-C63-D21-D83-L25

\footnotetext{
${ }^{*}$ Corresponding author: Institute of Economics, Scuola Superiore Sant'Anna, Piazza Martiri della Liberta' 33, I-56127, Pisa (Italy). E-mail address: gdosi<at>sssup.it

${ }^{\dagger}$ Institute of Economics, University of Campinas, Barao Geraldo, Campinas - SP (BR), 13083970. E-mail address: marcelocpereira<at>uol.com.br.

${ }^{\ddagger}$ Institute of Economics, Scuola Superiore Sant’Anna, Piazza Martiri della Liberta’ 33, I-56127, Pisa (Italy). E-mail address: m.virgillito<at>sssup.it
} 


\section{Introduction}

Evolutionary theories of economic change have identified as the two main drivers of the dynamics of industries the mechanisms of market selection and of idiosyncratic learning by individual firms. In this perspective, the interplay between these two engines shapes the dynamics of entry-exit, the variations of market shares and collectively the patterns of change of industry-level variables such as average productivities. Learning entails a various processes of idiosyncratic innovation, imitation, changes in technique of production. Selection is the outcome of processes of market interaction where more competitive firms gain market shares at the expense of less competitive ones.

Three overlapping streams of analysis try to explain how such interplay operates. The first one, from the pioneering work by Ijiri and Simon, (1977) all the way to Bottazzi and Secchi, (2006), studies the result of both mechanisms in terms of the ensuing exploitation of "new business opportunities", captured by the stochastic process driving growth rates. A second stream (see Metcalfe, 1998), focuses on the processes of competition/selection represented by means of a replicator dynamics. Finally, Schumpeterian evolutionary models unpack the two drivers distinguishing between the idiosyncratic processes of change in the techniques of production and the dynamic of differential growth driven by heterogeneous profitabilities and the ensuing rates of investment (Nelson and Winter, 1982) or by an explicit replicator dynamics (Silverberg et al., 1988, Dosi et al., 1995).

Whatever the analytical perspective, the purpose here is to further investigate one of the key empirical regularities that emerge from the statistical analysis of the industrial dynamics (for a critical survey see Dosi, 2007), the "fat-tailed" distribution of firms' growth rates.

In Dosi et al., (2015) we implement a "bare bones", multi-firm, evolutionary simulation model, built upon the familiar replicator equation and a cumulative learning process, which turns out to be able to systematically reproduce several stylized facts characterizing the dynamics of industries, and in particular the fattailed distributions of growth rates. However, the evaluation of the robustness of this result is done there by the usual (restricted scope) sensitivity analysis, testing across different learning regimes a limited sample of interesting points in the parameters space of the model. Under this scenario it is not possible to guarantee that the expected results would hold true for the entire range of variation of each parameter, in particular when more than one parameter is changed at the same time (Saltelli and Annoni, 2010), sometimes in combinations that may not even hold economic sense.

Global scope sensitivity analysis of high-dimensional, non-linear simulation mod- 
els has been a theoretical and - more so - a practical challenge for a long time. Advancements in both statistical analytical frameworks and computer power have gradually addressed this issue over the past two decades, starting in engineering and the natural sciences, but now also applied in the social sciences. Building on what in the field is called meta-modeling, design of experiments and variance-based decomposition, in this work we investigate how robust the fat tail "ubiquitousness" feature is in our bare-bones model with regard to a global exploration of the parameters space.

In what follows, we apply the Kriging meta-modeling methodology to represent our model by a mathematically tractable approximation. Kriging is an interpolation method that under fairly general assumptions provides the best linear unbiased predictors for the response of more complex, possibly non-linear, typically computer simulation models. The kriging meta-model is estimated from a set of observations (from the original model) carefully picked using a near-orthogonal Latin hypercube design of experiments. This approach minimizes the required number of samples and allows for high computational efficiency without impacting on the goodness-offit of the meta-model. Finally, the fitted meta-model is used together with Sobol decomposition to perform a variance-based, global sensitivity analysis of the original model on all of its parameters. The process allows for a genuinely simultaneous analysis of all parameters across the entire relevant parameters space while trying to deal with both non-linear and non-additive systems.

\section{Empirical and theoretical points of departure}

Firms grow and decline by relatively lumpy jumps which cannot be accounted by the cumulation of small, "atom-less", independent shocks. Rather "big" episodes of expansion and contraction are relatively frequent. More technically, this is revealed by fat tail distributions of growth rates. A typical empirical finding is illustrated in Figure 1. The pattern applies across different levels of sectoral disaggregation, across countries, over different historical periods for which there are available data and it is robust to different measures of growth, e.g., in terms of sales, value added or employment (for details see Bottazzi et al., 2002, Bottazzi and Secchi, 2006 and Dosi, 2007). What could be determining such property?

In general, such fat-tailed distributions are a powerful evidence of some underlying correlation mechanism. Intuitively, new plants arrive or disappear in their entirety, and, somewhat similarly, novel technological and competitive opportunities tend to arrive in "packages" of different "sizes" (i.e., economic importance). In turn, firm-specific increasing returns in business opportunities, as shown by Bottazzi and Secchi, (2003) are a source of such correlations. In particular, the latter build upon 


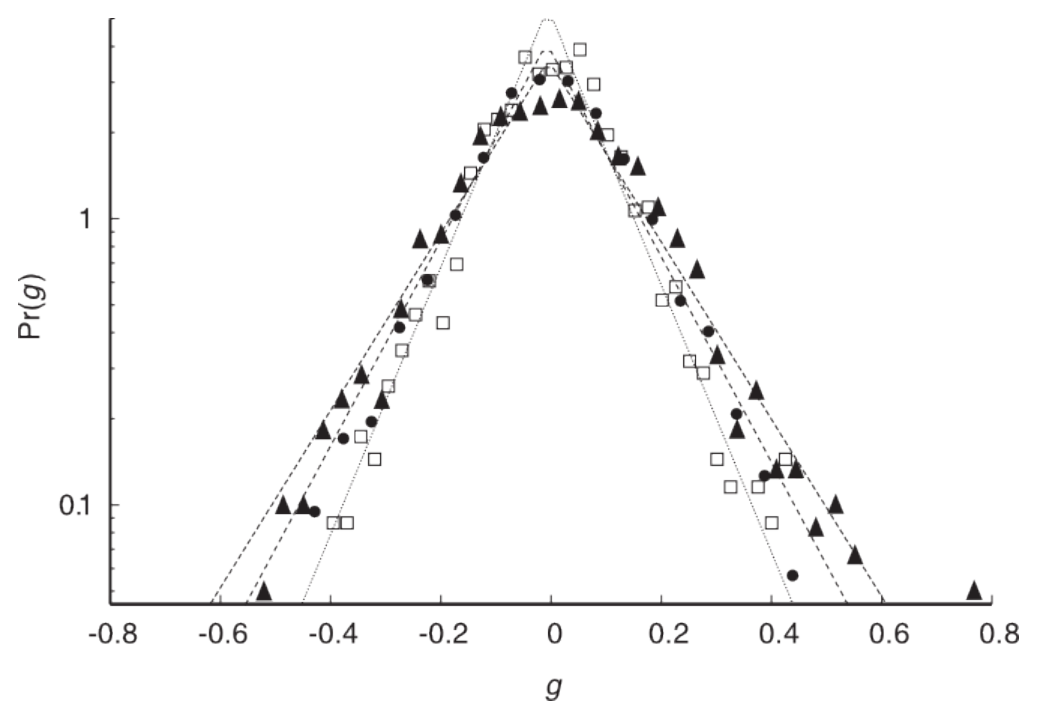

Figure 1: Tent shaped size growth rates (Italy, Istat Micro.1 data). Source: Bottazzi and Secchi, (2006).

the "island" model by Ijiri and Simon, (1977) and explore the hypothesis of a pathdependent exploitation of business opportunity via a Polya Urn scheme, wherein in each period "success breeds success". This cumulative process does account for the emergence of fat tails.

In Dosi et al., (2015) we show, by means of a simple simulation model, that competitive interactions induce correlation in the entry-growth-exit dynamics of firms entailing the absence of Gaussian distributions of growth rates. Fat tails emerge independently of the competition regime and the distributional forms of the innovation shocks. Moreover, under the most empirical-friendly regime - which assumes some level of cumulative learning - the distribution of growth rates produced by the model were close to the Laplace distribution, as such a particular instance of fat-tailed distribution quite akin to the shape of Figure 1. To further test the robustness of the results obtained in that work, three methodological tools are proposed namely, in sequence: design of experiments selection (sampling), meta-modeling and variance-based global sensitivity analysis. The challenge is to overcome the technical and computational constraints entangled in the original model - in particular, non-linearity and non-additivity (for thorough overviews, see Cioppa and Lucas, 2007, Rasmussen and Williams, 2006 and Saltelli et al., 2008).

As numerical simulation has become a standard tool in the natural sciences, and more recently also in the social sciences, the challenge of parsimoniously evaluate their results has become a paramount one. As models grow in size and complexity, the "naive" efforts to accurately explore their behavior by "brute force" or "one factor at a time" approaches quickly show their severe limitations in terms of com- 
putational times required and the poor expected accuracy (Helton et al., 2006, Saltelli and Annoni, 2010). Hence, the search for mathematically "well behaved" approximations of the inner relations of the original simulated model, frequently denominated surrogate models or meta-models, has become increasingly common (Kleijnen and Sargent, 2000, Roustant et al., 2012). The meta-model is a simplified version of the original model that can be more parsimoniously explored - at reasonable computational costs - to evaluate the effect of inputs/parameters on the latter and (likely) also on the former. Usual techniques employed for meta-modeling are linear polynomial regressions, neural networks, splines and Kriging.

Kriging (or Gaussian process regression), in particular, is suggested to be a simple but efficient method for investigating the behavior of simulation models (Van Beers and Kleijnen, 2004). Kriging meta-models came originally from the geosciences (Krige, 1951, Matheron, 1963). In essence, it is a Bayesian-based, spatial interpolation method for the prediction of a system response on unknown points based on the knowledge of such response on a set of previously known ones (the observations) to fit a real-valued random field. Under some set of assumptions, the Kriging meta-model can be shown to provide the best linear unbiased prediction for such points (Roustant et al., 2012). The intuition behind it is that the original model response for the unknown points can be predicted by a linear combination of the responses at the closest known points, similarly to an ordinary multivariate linear regression, but taking the spatial information into consideration, in a Bayesian framework. Recent advancements extended the technique, by removing the original assumption that the samples are noise free, made Kriging particularly convenient for the meta-modeling of stochastic computer experiments (Rasmussen and Williams, 2006).

Kriging, as any meta-modeling methodology, is based on the statistical estimation of coefficients for specific functional forms (decribed in Section 4) based on data observed from the original system or model. Kriging meta-models are frequently estimated over a near-orthogonal Latin hypercube (NOLH) design of experiments ${ }^{1}$ (McKay et al., 2000, and nearer to our concerns here Salle and Murat, 2014). The NOLH is a statistical technique for the generation of plausible sets of points from multidimensional parameter distributions with good space-filling properties (Cioppa and Lucas, 2007). It significantly improves the efficiency of the sampling process in comparison to traditional Monte Carlo approaches, requiring far smaller samples and much less (computer) time - to the proper estimation of meta-model coefficients (Helton et al., 2006, Iooss et al., 2010).

\footnotetext{
${ }^{1}$ In the present case it may be more appropriate to call the choice of the sampling points in the parameters space as quasi-experiment, as the conditions imposed for selecting the observations for the sample are specified by the NOLH.
} 
Sensitivity analysis (SA) aims at "studying how uncertainty in the output of a model (numerical or otherwise) can be apportioned to different sources of uncertainty in the model input" (Saltelli et al., 2008). Due to the high computational costs of performing traditional SA on the original model (e.g., ANOVA), authors like Kleijnen and Sargent, (2000), Jeong et al., (2005) or Wang and Shan, (2007) argue that the meta-model SA can be a reliable proxy for the original model behavior. Building on this assumption, one can propose the global SA analysis of the Kriging meta-model - as we attempt here - to evaluate the response of the original model over the entire parametric space, providing measurements of the direct and the interaction effects of each parameter. Following Saltelli et al., (2000), for the present analysis we selected a Sobol decomposition form of variance-based global SA analysis. It decomposes the variance of a given output variable of the model in terms of the contributions of each input (parameter) variance, both individually and in interaction with every other input by means of Fourier transformations. This method is particularly attractive because it evaluates sensitivity across the whole parametric space - it is a global approach - and allows for the independent SA analysis of multiple output models while being able to deal with non-linear and non-additive models (Saltelli and Annoni, 2010).

The approach proposed here has proved insightful for the analysis of non-linear simulation models, including economic ones: see Salle and Murat, $(2014)^{2}$ on two classic models and Bargigli et al., (2016) for an application to an agent-based model of financial markets.

\section{The original simulation model}

The model of departure, extensively presented and discussed in Dosi et al., (2015), represents the learning process by means of a multiplicative stochastic process upon firms productivities $a_{i} \in \mathbb{R}^{+}, i=1, \ldots, N$, in time $t=1, \ldots, T$ :

$$
a_{i}(t)=a_{i}(t-1)\left\{1+\max \left[0, \theta_{i}(t)\right]\right\}
$$

where $\theta_{i} \in \mathbb{R}^{+}$are realizations of a sequence of random variables $\{\Theta\}_{i=1}^{N}, N$ is the number of firms in the market and $T$ is the number of simulation time steps. Such dynamics is meant to capture the idiosyncratic accumulation of capabilities within each firm (more in Dosi et al., 2000). The process is a multiplicative random walk with drift: the multiplicative nature is well in tune with the evidence on productivity dynamics under the further assumption that if a firm draws a negative $\theta_{i}$, it will stick

\footnotetext{
${ }^{2}$ Here, we closely follow, whenever possible, the analytical framework employed by those authors and refer the readers to their paper for additional details and references.
} 
to its previous technique (negative shocks in normal times are quite unreasonable!), meaning that the lower bound for the support of the shocks $\theta_{i}$ distribution is zero.

In Dosi et al., (2015) we experiment with different learning regimes. Different specifications were tested for $\theta_{i}$. In particular, we focus here on the regime called Schumpeter Mark II, after the characterization of the "second Schumpeter" (Schumpeter, 1947). In this specification, incumbents do not only learn, but do it in a cumulative way so that a productivity shock in any firm is scaled by its extant relative competitiveness:

$$
\begin{gathered}
\theta_{i}(t)=\min \left[\pi_{i}(t)\left(\frac{a_{i}(t-1)}{\bar{a}(t-1)}\right)^{\gamma}, \mu_{\max }\right], \\
\bar{a}(t-1)=\sum_{i} a_{i}(t-1) s_{i}(t-1)
\end{gathered}
$$

where $\gamma \in \mathbb{R}^{+}$is a parameter, $0<s_{i}(t) \leq 1$ is the market share of firm $i$ which changes as a function of the ratio of the firm's productivity (or "competitiveness") $a_{i}(t)$ to the weighted average of the industry $\bar{a}(t)$ and $\pi_{i} \in \mathbb{R}$ is a random drawn from a set of possible alternative distributions, being a rescaled Beta distribution the default case. ${ }^{3} \pi_{i}(t)$ distribution has average equal to $\mu \in \mathbb{R}^{+} . \theta_{i}(t)$ is limited by an upper bound $\mu_{\max } \in \mathbb{R}^{+}$, based on the empirical evidence on the existence of a finite limit to the innovation shocks amplitude.

Competitive interactions are captured by a "quasi-replicator" dynamics:

$$
\begin{aligned}
\Delta s_{i}(t, t-1) & =A s_{i}(t-1)\left(\frac{a_{i}(t)}{\bar{a}(t)}-1\right), \\
\bar{a}(t) & =\sum_{i} a_{i}(t) s_{i}(t-1)
\end{aligned}
$$

where $A \in \mathbb{R}^{+}$is an elasticity parameter that captures the intensity of the selection exerted by the market, in terms of market share dynamics and, indirectly, of mortality of low competitiveness firms. $a_{i}(t)$ is calculated over the lagged market shares $s_{i}(t-1)$ for temporal consistency.

Finally, firms with market share $s_{i}(t)$ lower than the parameter $0<s_{\min }<1$ exit the market ("die") and market shares are accordingly recomputed. We assume that entry of new firms occurs (inverse) proportionally to the number of "surviving" incumbents in the market:

$$
E(t)=N-I(t-1)
$$

where $E(t): \mathbb{N} \rightarrow \mathbb{N}$ defines the number of entrants at time $t, I(t-1) \in \mathbb{N}$ is the number of incumbents in the previous period and $N$ is defined as above. The empirical evidence supports the idea that there is a rough proportionality between entry

\footnotetext{
${ }^{3}$ The rescaled Beta distribution was preferred because of its superior flexibility in terms of parametrization and the bounded support. Other than Beta, Laplace and Gaussian, Log-normal and Poisson distributions were also tested in Dosi et al., (2015). Different distributions did not qualitatively affect the results.
} 
and exit, thus, in the simplest version of the model, we assume a constant number of firms with the number of dying firms offset by an equal number of entrants.

The productivity of entrant $j$ follows a process similar to Eq. (1) but applied to the average productivity of the industry at the moment of entry, whose stochastic component $\theta_{j}$ is again a random drawn from the applicable distribution for $\pi_{i}$ as in Eq. 2 (under $\gamma=0)$ :

$$
a_{j}(t)=\bar{a}(t)\left(1+\theta_{j}(t)\right)
$$

being $\bar{a}(t)$ calculated as in Eq. (3). Of course, here $\theta_{j}(t)$ can get negative values. Indeed, the location of the mass of the distribution - over negative or positive shocks - captures barriers to learning by the entrant or, conversely, the "advantage of newness". Entrant initial size is constant at $s_{j}\left(t_{0}\right)=1 / N$.

Table 1 summarizes all the model and the alternative distributions parameter settings - our "default" configuration for the model - as well the remaining model simulation setup. ${ }^{4}$ Because of the stochastic component in $\theta_{i}$, the model outputs are non-deterministic, so the aggregated results must evaluated in terms of the mean and the variance of the output variables over a Monte Carlo (MC) experiment. It is executed by a given number of model runs under different seeds for the random number generator but with the same parameters configuration. Considering the measured variance of the relevant output variables and a target significance level of $5 \%$, a MC sample of 50 runs was determined as sufficient to fully qualify the model results.

\subsection{Timeline of events}

- There are $N$ initial firms at time $t=1$ with equal productivity and market share.

- At the beginning of each period firms learn according to Eq. (1).

- Firms acquire or lose market share, according to the replicator in Eq. (3).

- Firms exit the market according to the rule $s_{i}(t)<s_{\text {min }}$.

- The number and competitiveness of entrants are determined as in Eqs. (4) and (5).

- After entry market shares of incumbents are adjusted proportionally.

\footnotetext{
${ }^{4}$ The simulation model is coded in $\mathrm{C}++$ and it is run inside the LSD simulation platform (Valente, 2014) which is also employed for the NOLH sampling procedure, as explained below.
} 


\begin{tabular}{lc}
\hline & VALUE \\
\hline$A$ & 1.0 \\
$N$ & 150 \\
$s_{\text {min }}$ & 0.001 \\
$\gamma$ & 1.0 \\
$\mu$ & 0.05 \\
$\mu_{\text {max }}$ & 0.2 \\
Beta shocks $\left(\beta_{\alpha}, \beta_{\beta}, \beta_{\min }, \beta_{\max }\right)$ & $(1.0,5.0,0.0,0.3)$ \\
Laplace shocks $\left(\alpha_{1}, \alpha_{2}\right)$ & $(0.01,0.015)$ \\
Gaussian shocks $(\sigma)$ & $(0.8)$ \\
Initial productivity $\left(a_{i}(1)\right)$ & 1.0 \\
Initial market share $\left(s_{i}\left(t_{0}\right)\right)$ & $1 / N$ \\
\hline Number of time steps $(T)$ & 200 \\
Number of MC runs & 50 \\
\hline
\end{tabular}

Table 1: Parameters and simulation default settings.

\subsection{Firm growth rates distribution}

The growth rate of firm sizes is defined as:

$$
g_{i}(t)=\log s_{i}(t)-\log s_{i}(t-1)
$$

where the market share $s_{i}$ is used as a proxy for the firm size.

In order to test the robustness of the results to the shocks specification, in what follows we experiment with three alternative distributions for the innovation shocks, namely rescaled Beta, Laplace and Gaussian, configured with the parameters set forth in Table 1. Figure 2 shows the simulation results for the three distributions. The departure from (log) normality and the emergence of fat tails is rather striking, independently of the shape of the micro-shocks distribution.

To measure how "fat" the tails of the distributions are, we estimate the $b$ parameter of a symmetric Subbotin distribution:

$$
f_{S}(x)=\frac{1}{2 a b^{1 / b} \Gamma(1 / b+1)} e^{-\frac{1}{b}\left|\frac{x-\mu}{a}\right|^{b}}
$$

defined by the parameters $m, a$ and $b$, wherein $m$ is a location measure (the median), $a$ is the scale parameter and $b$ captures the "fatness" of the tails. Such a distribution, according to the value of the parameter $b$, can yield a Gaussian distribution, if $b=2$, or a Laplace distribution, if $b=1$, among other results for different values of $b$. Estimates of the Subbotin distribution $b$ parameter are also presented in Figure $2{ }^{5}$

\footnotetext{
${ }^{5}$ Subbotin parameters estimation is performed by the maximum-likelihood method using the Subbotools package (Bottazzi, 2014).
} 


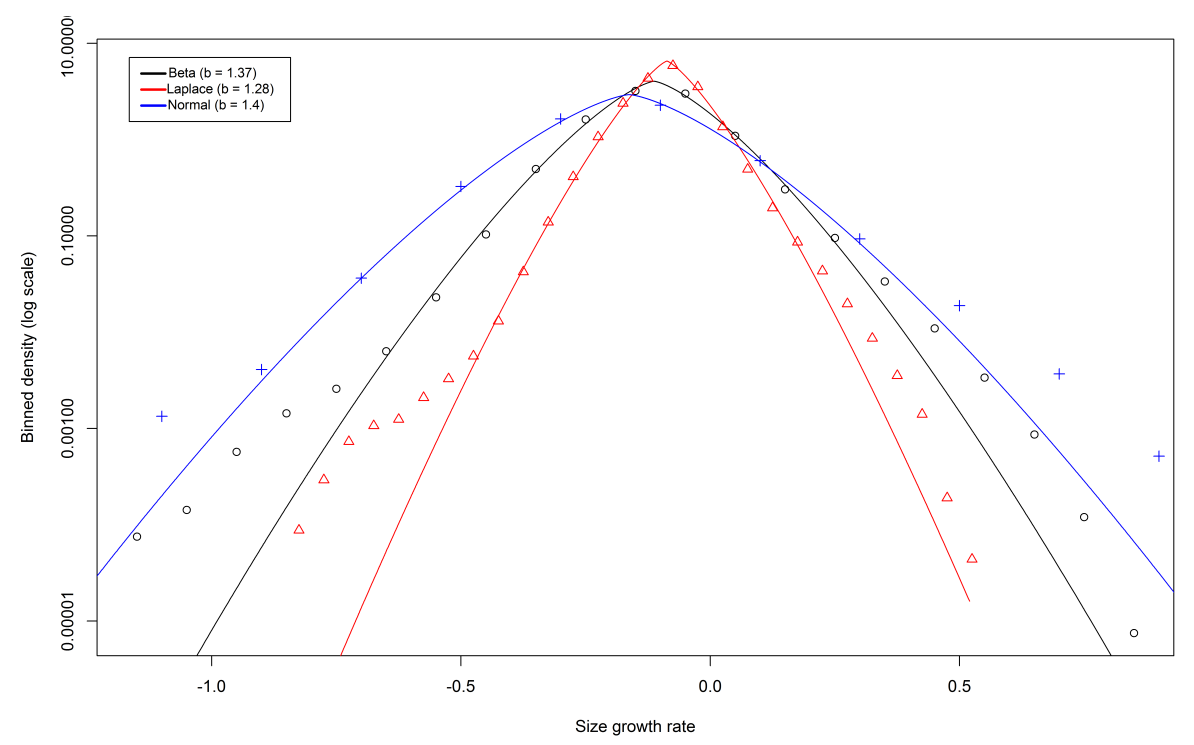

Figure 2: Firm growth rates under different distributions of innovation shocks.

Across the three distributions, the value of the $b$ parameter is always significantly smaller than 2 (the normality case).

\section{Exploring the robustness of the fat tails}

As the results presented above suggest the presence of distributions "fatter" than Gaussian across configurations, further inquiry on the generality of these findings seems important. A first step in this direction is performed in Dosi et al., (2015) for some alternative parameter settings. However, even if this approach is still the current standard for most computer simulations analyses, it is likely not sufficient for non-linear, non-additive setups, as convincingly demonstrated by Saltelli and Annoni, 2010. Given the current model has a clear non-linear nature, the adoption of more general investigation methods seems recommended. To address the task at hand we propose the application of a numerical analysis procedure based on a Bayesian framework, as discussed in Section 2. The proposed steps are:

1. NOLH DoE: construct an appropriate design of experiments (DoE) performing efficient sampling via the NOLH approach.

2. Kriging meta-modeling: estimate and choose among alternative Kriging meta-model specifications.

3. Global sensitivity analysis: analyze the meta-model sensitivity to each parameter of the model using Sobol (variance) decomposition. 
4. Response surface: graphically map the meta-model response surface $(2 \mathrm{D}$ and 3D) over the more relevant parameters and identify critical areas.

In a nutshell, the Kriging meta-model $Y$ is intended to predict the response of a given (scalar) output variable $y$ of the original simulation model: ${ }^{6}$

$$
Y(\mathbf{x})=\lambda(\mathbf{x})+\delta(\mathbf{x})
$$

where $\mathbf{x} \in D$ is a vector representing any point in the parametric space domain $D \subset$ $\mathbb{R}^{k}$, being $x_{1}, \ldots, x_{k} \in \mathbb{R}$ the $k \geq 1$ original model parameters and $\lambda(\mathbf{x}): \mathbb{R}^{k} \rightarrow \mathbb{R}$, a function representing the global trend of the meta-model $Y$ under the general form:

$$
\lambda(\mathbf{x})=\sum_{i=1}^{l} \beta_{i} f_{i}(\mathbf{x}), \quad l \geq 1
$$

being $f_{i}(\mathbf{x}): \mathbb{R}^{k} \rightarrow \mathbb{R}$ fixed arbitrary functions and $\beta_{1}, \ldots, \beta_{l}$ the $l$ coefficients to be estimated from the sampled response of the original model over the image of $y$. The trend function $\lambda$ is assumed here, for simplicity, to be a polynomial of order $l-1$, more specifically of order zero ( $\beta_{1}$ is the trend intercept) or one $\left(\beta_{2}\right.$ is the trend line inclination). This is usually enough to fit even complex response surfaces when coupled with an appropriate design of experiment (DoE) sampling technique. ${ }^{7}$

In Eq. (8), $\delta(\mathbf{x}): \mathbb{R}^{k} \rightarrow \mathbb{R}$ models the stochastic process representing the local deviations from the global trend component $\lambda . \delta$ is assumed second-order stationary with zero mean and covariance matrix $\tau^{2} R$ (to be estimated), where $\tau^{2}$ is a scale parameter and $R$ is a $n \times n$ matrix ( $n$ is the number of observations) whose $(i, j)$ element represents the correlation among $\delta\left(\mathbf{x}_{i}\right)$ and $\delta\left(\mathbf{x}_{j}\right), \mathbf{x}_{i}, \mathbf{x}_{j} \in D, i, j=$ $1, \ldots, n$. The Kriging meta-model assumes a close correspondence between this and the correlation across $y\left(\mathbf{x}_{i}\right)$ and $y\left(\mathbf{x}_{j}\right)$ in the original model. Different specifications can be used for the correlation function, according to the characteristics of the $y$ surface. For example, one of the simplest candidates is the power exponential function:

$$
\operatorname{corr}\left(\delta\left(\mathbf{x}_{i}\right), \delta\left(\mathbf{x}_{j}\right)\right)=\exp \left[-\left(\sum_{g=1}^{k} \psi_{g}\left|x_{g, i}-x_{g, j}\right|\right)^{p}\right]
$$

where $x_{g, i}$ denotes the value of parameter $x_{g}$ at the point $\mathbf{x}_{i}, \psi_{1}, \ldots, \psi_{k}>0$ are the $k$ coefficients to be estimated and $0<p \leq 2$ is the power parameter ( $p=1$ for the ordinary exponential correlation function). They quantify the relative weight of parameter $x_{g}, g=1, \ldots, k$, on the overall correlation between $\delta\left(\mathbf{x}_{i}\right)$ and $\delta\left(\mathbf{x}_{j}\right)$

\footnotetext{
${ }^{6}$ In this section we loosely follow the formalization proposed by Roustant et al., (2012) and Salle and Murat, (2014).

${ }^{7}$ Higher order polynomials were evaluated but systematically produced meta-models with worse fitting to the original model, even when more samples are added to the DoE.
} 
and, hopefully, among $y\left(\mathbf{x}_{i}\right)$ and $y\left(\mathbf{x}_{j}\right)$. Notice that a higher $\psi_{g}$ represents a smaller influence of parameter $x_{g}$ over $\delta .^{8}$

Therefore, the Kriging meta-model requires $l+k+1$ coefficients to be estimated over the $n$ observations selected by an appropriate design of experiments (DoE). As discussed before, $l=1$ or 2 is adopted. $k$ is determined by the number of parameters of the original model that are being evaluated in the sensitivity analysis, so it is dependent on the specification of the innovation shocks (rescaled Beta, Laplace or Gaussian). The original simulation model has four base parameters: $A$ (replicator sensitivity), $N$ (number of firms), $s_{\min }$ (the market share below which a firm exits the market) and $\gamma$ (learning cumulativity). The alternative shocks distributions have two common parameters: $\mu$ and $\mu_{\max }$ (the average shock size and the upper support limit). Additionally, rescaled Beta distribution requires $\beta_{\alpha}, \beta_{\beta}$ (shape parameters), $\beta_{\min }$ and $\beta_{\max }$ (support limits), Laplace needs $\alpha_{1}$ and $\alpha_{2}$ (shape parameters) and Gaussian, $\sigma$ (standard deviation), leading to a total of $k=10,7$ and 6 parameters to test, respectively.

In practical terms, we constrained the experimental domain to ranges of the parameters that are empirically reasonable and respect minimal technical restrictions of the original model, ${ }^{9}$ according to Table 2 . The output variable tested $(y)$ is the selected "fat-taildness" measure of the distribution of firms' growth rates $(b)$ on the original model. Therefore, $y=b$ is estimated by the maximum-likelihood fit for the $b$ shape parameter of a Subbotin distribution (as defined above).

Three designs of experiments are created to evaluate each innovation shocks specification. We use the rescaled Beta distributed shocks case to present the results more extensively. The other cases, conversely, will be presented in a more concise form. For the rescaled Beta $(k=10)$ and the Laplace $(k=7)$ configurations, DoE's with $n=33$ samples are created. For the Gaussian $(k=6)$ case, while $n=17$ is usually considered an adequate DoE size, we also select $n=33$ because both the $Q^{2}$ and the RMSE goodness-of-fit measures (see below) perform much worse under the smaller DoE when compared to the other two cases. The near-orthogonal Latin hypercube (NOLH) DoE's are constructed according to the recommendations provided by Cioppa and Lucas, (2007). Yet, for the external validation procedures (see below), 10 additional random samples are generated for each DoE. Because of the stochastic nature of the original model, each point $\mathbf{x}_{i}, i=1, \ldots, n$ in the parametric space is computed over $m=50$ simulation runs using different seeds for

\footnotetext{
${ }^{8}$ Definitions for other correlation function alternatives can be found in Roustant et al., 2012.

${ }^{9}$ The technical feasibility criterion adopted was the minimally "normal" operation of the market, measured by the survival of at least two firms during the majority of simulation time steps. Also, some of the parameters' test ranges limit, in practice, the possible ranges of variation for other parameters (e.g., the distribution average $\mu$ must be lower than the upper support of distributions $\left.\mu_{\max }\right)$.
} 


\begin{tabular}{llcc}
\hline Parameter & & Minimum & Maximum \\
\hline Base model & $A$ & 0.2 & 5.0 \\
& $N$ & 50 & 350 \\
& $s_{\min }$ & 0.0001 & 0.0015 \\
& $\gamma$ & 0.2 & 5.0 \\
& $\mu$ & 0.015 & 0.15 \\
Beta shocks & $\mu_{\max }$ & 0.2 & 1.0 \\
& $\beta_{\alpha}$ & 1.0 & 3.0 \\
& $\beta_{\beta}$ & 3.0 & 10.0 \\
& $\beta_{\min }$ & 0.0 & 0.1 \\
& $\beta_{\max }$ & 0.2 & 1.0 \\
Laplace shocks & $\alpha_{1}$ & 0.005 & 0.05 \\
& $\alpha_{2}$ & 0.005 & 0.05 \\
Gaussian shocks & $\sigma$ & 0.2 & 2.0 \\
\hline
\end{tabular}

Table 2: Parameters experimental space domain $D$.

the pseudo-random number generator. The resulting $y\left(\mathbf{x}_{i}\right)=b_{i}\left(\mathbf{x}_{i}\right)$ is evaluated by the mean of the observed $\tilde{b}_{i, m}$ across the $m$ runs and its variance is used to specify the noise in - or the weight of - each point of the DoE in the estimation of $Y .{ }^{10}$

As discussed above, adequate trend and correlation functions must be selected - in Bayesian terms, they are the required priors - for estimation of the Kriging meta-model. To choose among potential candidates, we perform an evaluation of the goodness-of-fit (of the meta-model to the original model response surface) based on both cross (in-sample) and external (out-of-sample) validation, as suggested by Salle and Murat, (2014). Cross validation is performed using the $Q^{2}$ predictivity coefficient (a proxy of conventional $R^{2}$ ). External validation is based on the root mean square error ( $R M S E)$ measure. The two criteria are usually compatible and for meta-model estimation we selected the function pair performing better according to both criteria (50:50\% weight). Results for the rescaled Beta case are presented in Table 3. The analysis was performed for the three cases but not included here as the general results are similar. The selected function pair for each case is presented next.

The estimated Kriging meta-models, according to Eqs. (8) - (10), are shown in Table $4 .{ }^{11}$ General meta-model fitting was good, as measured by both cross $Q^{2}$ and external $R M S E$ validations.

\footnotetext{
${ }^{10}$ Noise is used in the entire estimation process to evaluate observations. Samples under too much noise (sampling variance over 10 times the average) are discarded in the estimation process. Table 4 presents the effective number of observations used.

${ }^{11}$ The meta-model estimation and the following sensitivity analysis was performed using the DiceKriging, DiceOptim and DiceEval packages (Roustant et al., 2012, Dupuy et al., 2015) in R (R Core Team, 2016).
} 
Table 3: Comparison of alternative meta-model specifications (Beta shocks). Higher $Q^{2}$ and lower $R M S E$ values are better.

\begin{tabular}{crccccc}
\hline Validation & $f_{i}$ Trend & Matèrn 5/2 & Matèrn 3/2 & Gaussian & Exponential & Power exp. \\
\hline Cross & Constant & 0.2763 & 0.3073 & 0.2527 & 0.0793 & 0.2879 \\
$\left(Q^{2}\right)$ & Linear & 0.0000 & 0.0000 & 0.0000 & 0.0000 & 0.0000 \\
\hline External & Constant & 1.0249 & 1.0514 & 1.0015 & 1.2370 & 1.0179 \\
$(R M S E)$ & Linear & 1.0112 & 1.0112 & 1.0112 & 1.0112 & 1.0112 \\
\hline
\end{tabular}

Table 4: Kriging meta-model estimation.

\begin{tabular}{lccc}
\hline & BETA SHOCKS & LAPLACE SHOCKS & GAUSSIAN SHOCKS \\
\hline Trend function & constant & constant & constant \\
$\lambda($ intercept $)$ & 1.4649 & 1.4092 & 1.9603 \\
Correlation function & Power exp. & Matèrn $5 / 2$ & Matèrn $5 / 2$ \\
$\psi(A)$ & 3.5984 & 3.5630 & 3.2971 \\
$\psi(N)$ & 243.7065 & 101.5874 & 257.2181 \\
$\psi(s M i n)$ & 0.0005 & 0.0005 & 0.0010 \\
$\psi(\gamma)$ & 9.2324 & 2.0045 & 8.0193 \\
$\psi(\mu)$ & 0.2700 & 0.0966 & 0.1485 \\
$\psi\left(\mu_{\text {max }}\right)$ & 1.6000 & 1.6000 & 1.6000 \\
$\psi\left(\beta_{\alpha}\right)$ & 3.8186 & & \\
$\psi\left(\beta_{\beta}\right)$ & 10.4382 & & \\
$\psi\left(\beta_{\text {min }}\right)$ & 0.2000 & & \\
$\psi\left(\beta_{\text {max }}\right)$ & 1.4909 & & \\
$\psi\left(\alpha_{1}\right)$ & & & \\
$\psi\left(\alpha_{2}\right)$ & & 0.0872 & 3.6000 \\
$\psi(\sigma)$ & 1.0179 & 0.0900 & 0.6454 \\
\hline Cross validation $Q^{2}$ & 33 & & 0.4721 \\
External validation $R M S E$ & 10 & 32 & 32 \\
NOLH samples used $(n)$ & 0.2879 & 10 & 10 \\
External validation samples & & & \\
\hline
\end{tabular}




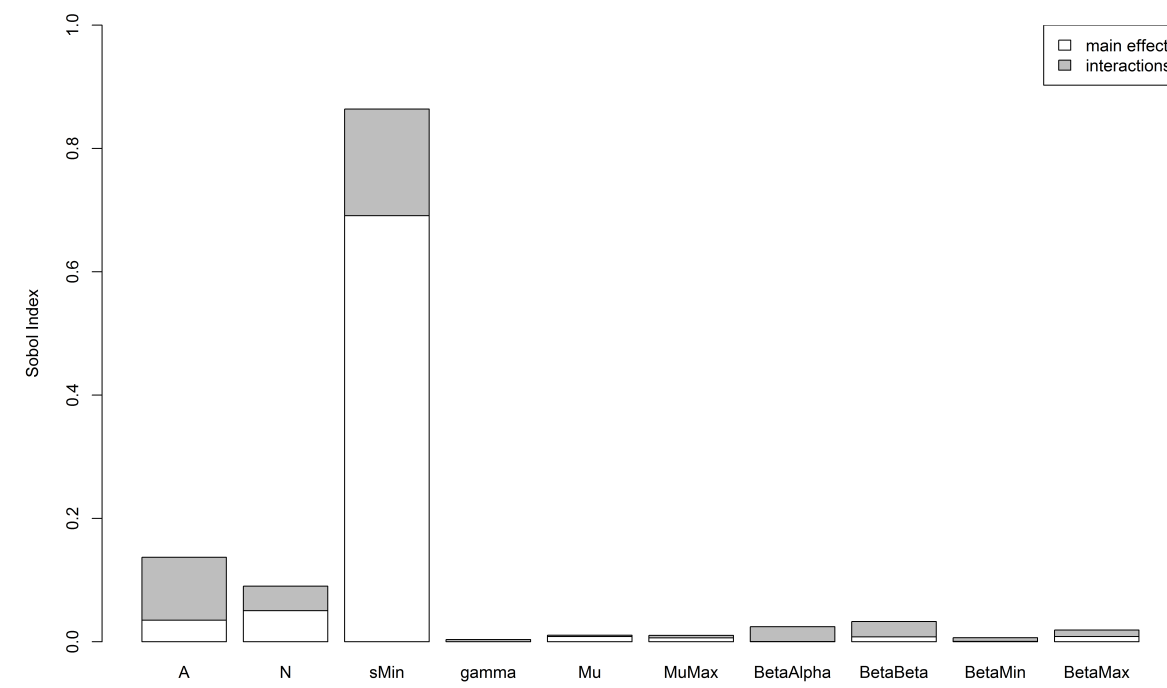

Figure 3: Sensitivity analysis of parameters effects on meta-model response (Beta shocks).

The magnitudes of the estimated $\psi$ coefficients provide a rough indication of the (inverse) importance of each parameter on the variation of the Subbotin's $b$ shape parameter of the firm's growth rates distribution. However, a more refined analysis is proposed in Figure 3. There we present the results of the Sobol (variance) decomposition procedure, as proposed by Saltelli et al., (2000), comprised by the individual and the interaction effects of each parameter on the variance of $Y$ - and, likely, also of $y$. Even considering the significantly different specifications, results are reasonably similar among alternative shocks configurations. Figures 3 and 5 ([a] and $[\mathrm{d}])$ show the sensitivity analysis results. Unexpectedly, the $s_{\text {min }}$ parameter is the most influential in the three cases. Considering the direct effects, in the rescaled Beta case (estimated under a power exponential correlation function as per Eq. (10)) $s_{\min }$ accounts for more than $80 \%$ of the variance of $b$, while in the Laplace and the Gaussian cases (using a Matèrn $5 / 2$ covariance kernel) ${ }^{12}$ this influence is little under 60-50\%, respectively. The next relevant parameters are $A$ and $N$ for Beta (around $10 \%$ each), Laplace (20-40\% respectively) and Gaussian (30\% each). $\mu$ is relevant for Laplace and Gaussian (25-15\% respectively). Only in the Laplace case, $\gamma$ is also important (about 25\%) but mainly in interaction with the other parameters. $\mu_{\max }$ and all the distribution-specific parameters are relatively unimportant for the meta-model output.

\footnotetext{
${ }^{12}$ The Matèrn correlation function - the Fourier transform of the Student distribution density function - in its $5 / 2$ formulation can be specified as (Rasmussen and Williams, 2006):

$$
\operatorname{corr}\left(\delta\left(\mathbf{x}_{i}\right), \delta\left(\mathbf{x}_{j}\right)\right)=\left(1+\sqrt{5} h+\frac{5}{3} h^{2}\right) \exp (-\sqrt{5} h), \quad h=\sum_{g=1}^{k} \psi_{g}\left|x_{g, i}-x_{g, j}\right|
$$


Considering the three dominant parameters detected by the Sobol decomposition, Figure 4 shows the response surfaces of the rescaled Beta shocks meta-model for the full range of these parameters, as indicated in Table 2. The plots in the columns of Figure 4 represent the same response surface, the top one in a 3D representation and the one in the bottom using isolevel curves. In all plots, parameters $s_{\text {min }} \in[0.0001,0.0015]$ and $A \in[0.2,5]$ are explored over their entire variation ranges. The first and the last columns (Figure 4, plots [a], [c], [d] and [f]) show the response for the limit values of parameter $N \in[50,350]$, while the center column (plots [b] and [e]) depicts the default model setup (except for $s_{\min }$ and $A$ ). The round mark in the two plots represents the meta-model response at the full default settings, as per Table 1. The prediction of the meta-model for this particular point in the parameters space - which is not included in the DoE sample - is $\hat{b}=1.58$ while the "true" value from the original model is $b=1.37$, an error of $+15 \%$ wholly inside the expected $95 \%$ confidence interval for that point $(\epsilon= \pm 0.75) .{ }^{13}$ In particular, the default settings point is located at a level close to the global maximum of the response surface, around $\hat{b}=1.75$ (the minimum is at $\hat{b}=1$ ).

Coupled with the sensitivity analysis results, which show that $s_{\min }, A$ and $N$ are the only parameters significantly affecting the predicted $\hat{b}$, Figure 4 seems to corroborate the hypothesis that the model results are systematically fat-tailed, as can be inferred from the condition $\hat{b}<2$. However, considering the average $95 \%$ confidence interval $\bar{\epsilon}= \pm 0.68$ range for the meta-model response surface, it seems that still exists a region where we cannot discard the absence of fat-tails $(\hat{b} \geq 2)$ at the usual significance levels. Therefore, further analysis is required, this time focused in this particular area, representing a small portion of the parametric space where the meta-model resolution is not sufficient to completely specify the response of the original model. Considering the critical region only (approximated to $s_{\min } \in$ [0.0001, 0.001] and $A \in[0.2,3]$ ), a "brute force" Monte Carlo sampling approach is performed in the original model. Not surprisingly, out of 20 random observations, considered sufficient given the predicted smoothness of the investigated area at a $5 \%$ significance level, the sampled interval true response was in the range $[1.25,1.63]$, confirming that the meta-model predicted $\hat{b}$, in this particular region, is likely to overestimate the true value of the shape parameter $b$. In conclusion, it seems very probable that the true response surface of the original model is significantly under the $b=2$ limit over the entire explored parametric space for rescaled Beta innovation shocks.

Similar analysis is conducted for the Laplace and Gaussian innovation shocks

\footnotetext{
${ }^{13}$ Kriging predictions becomes more precise as the interpolated point gets closer to one of the DoE points, where the error of the model is always zero by construction - and vice versa - so $\epsilon$ is not constant.
} 
meta-models. The results are synthesized in Figure 5. The Laplace case is in the upper row (plots [a], [b] and [c]) which presents the Sobol decomposition sensitivity analysis, already discussed, and the surface response for the top two critical parameters $\left(s_{\min } \in[0.0001,0.0015]\right.$ and $\left.N \in[50,350]\right)$. Again, the meta-model predicted response with default settings is at a level $\hat{b}=1.51$, close to the global maximum at $\hat{b}=1.77$ and above the minimum at $\hat{b}=0.91$. The true value at the default point is $b=1.28$ and the prediction error is $+18 \%$, well under the $95 \%$ confidence interval $\epsilon= \pm 0.69$ in that point. As in the previous case, and despite the entire meta-model surface is substantially below the critical level $\hat{b}=2$, under the usual significance levels $(\bar{\epsilon}= \pm 0.77)$ there is a region of the surface where we cannot discard $b \geq 2$. However, once again the Monte Carlo exploration of this critical region on the original model, also seems to confirm the hypothesis of $b<2$ for the whole parametric space of the original model under Laplace innovation shocks.

Qualitatively close results come from the Gaussian meta-model. The dynamics of the meta-model here is driven by $s_{\min } \in[0.0001,0.0015]$ and $N \in[50,350]$. The produced response surface is slightly more rugged, as depicted in Figure 5 ([d], [e] and $[\mathrm{f}])$. The meta-model prediction for the default settings point is $\hat{b}=1.36$, well in between the surface's global minimum at $\hat{b}=0.98$ and the maximum at $\hat{b}=1.71$. The prediction error, in this case, is $-3 \%$ given the true $b=1.40$, easily inside the $95 \%$ confidence interval $\epsilon= \pm 0.74$. Again, considering the average $95 \%$ confidence interval $\bar{\epsilon}= \pm 0.47$ over the entire surface, there is a small region of the response surface (the "hilltop" around $N<70$ and $s_{\min }>0.0010$ ) where it's not possible to reject the absence of fat tails. However, specific MC exploration in this area on the original model once more produced no points sitting close to the $b=2$ limit, confirming the meta-model predictions of $b<2$ for the entire region. 


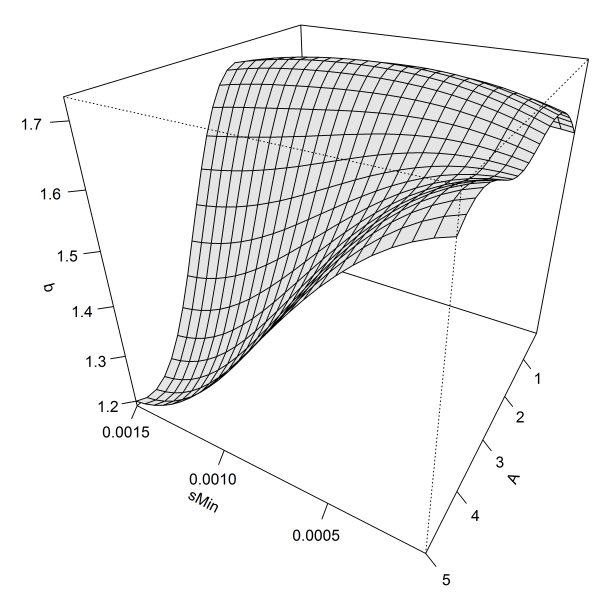

(a) $N=50$

$\infty$

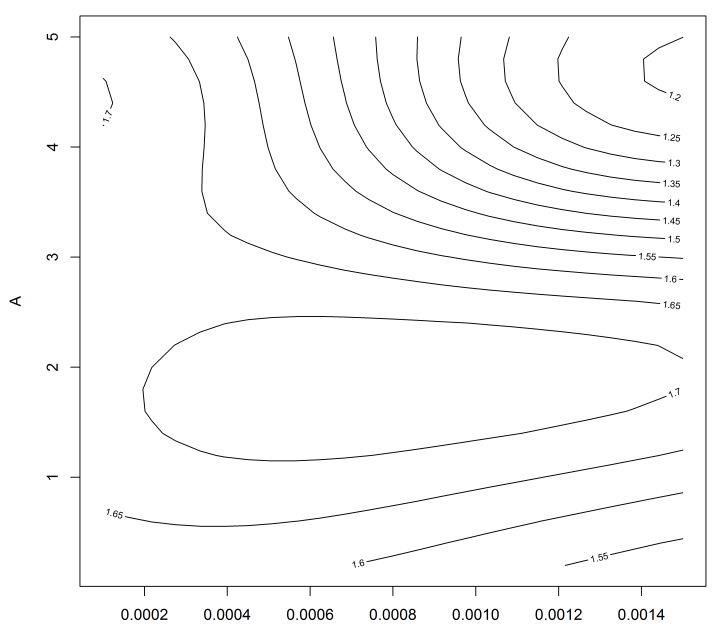

(d) $N=50$

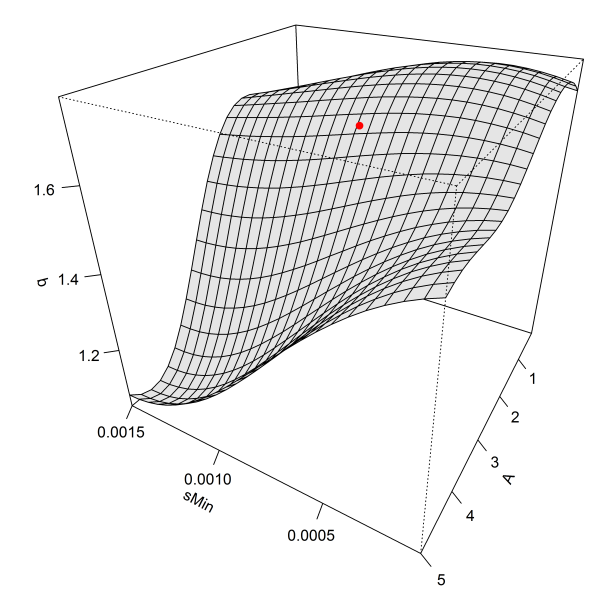

(b) $N=150$

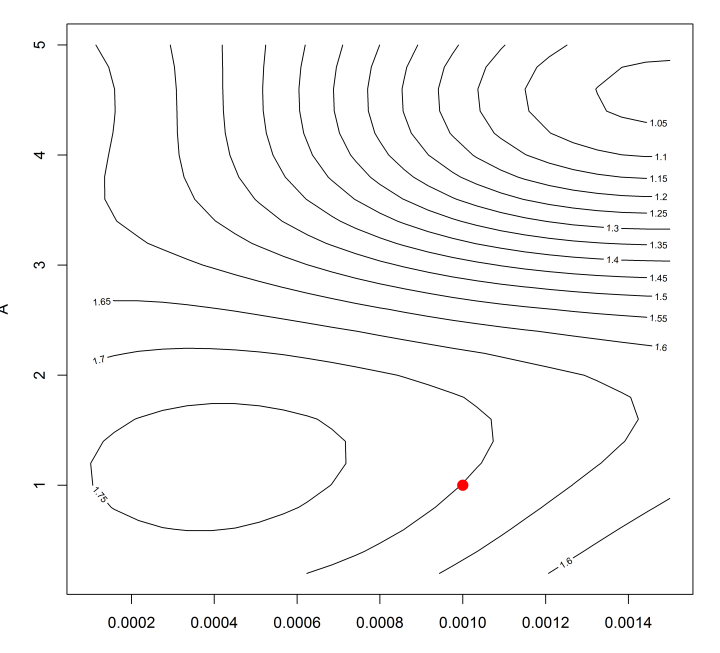

(e) $N=150$

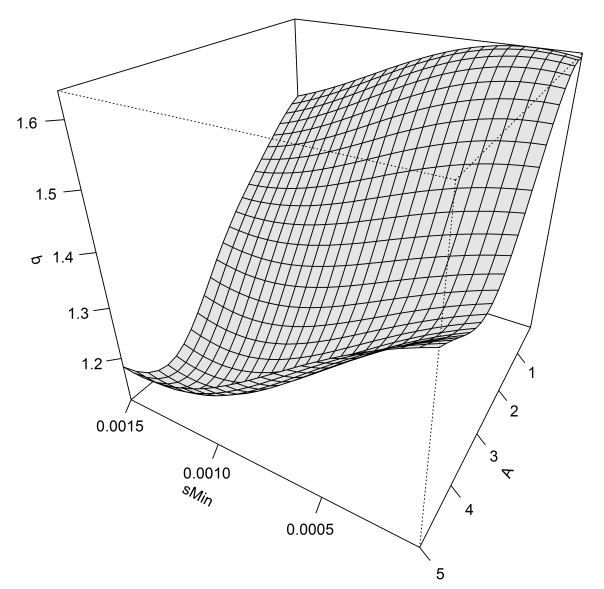

(c) $N=350$

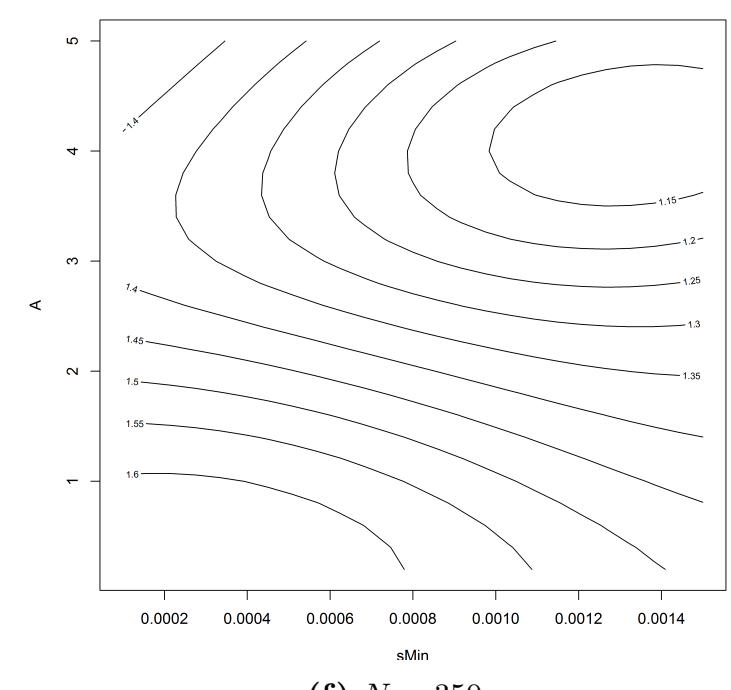

Figure 4: Response surfaces - Beta shocks. All remaining parameters set at default settings (round mark at default $s_{\min }$ and $A$ ). 


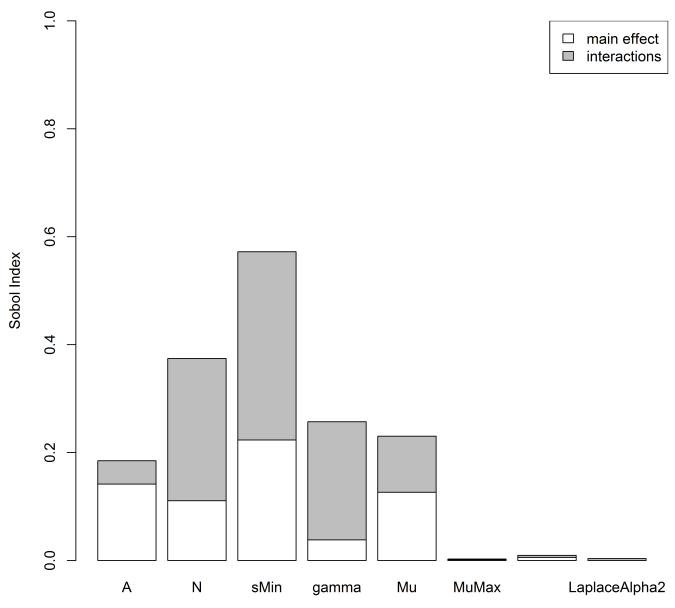

(a) Sensitivity analysis (Laplace)

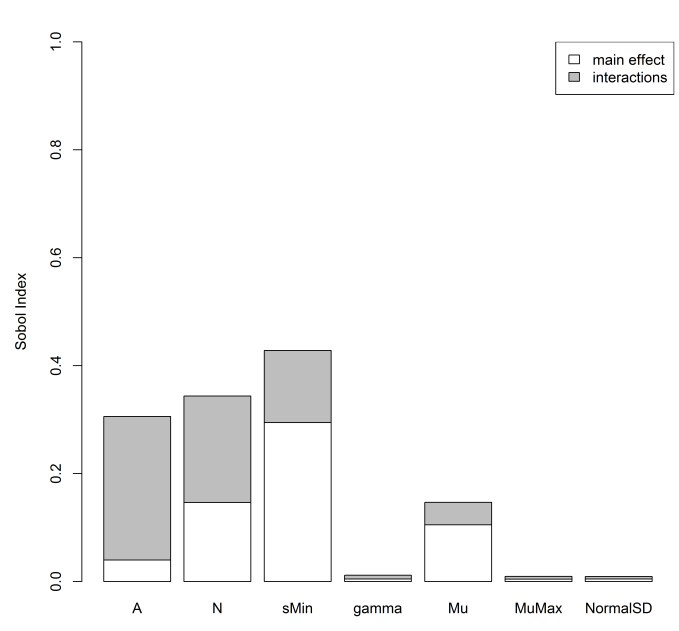

(d) Sensitivity analysis (Gaussian)

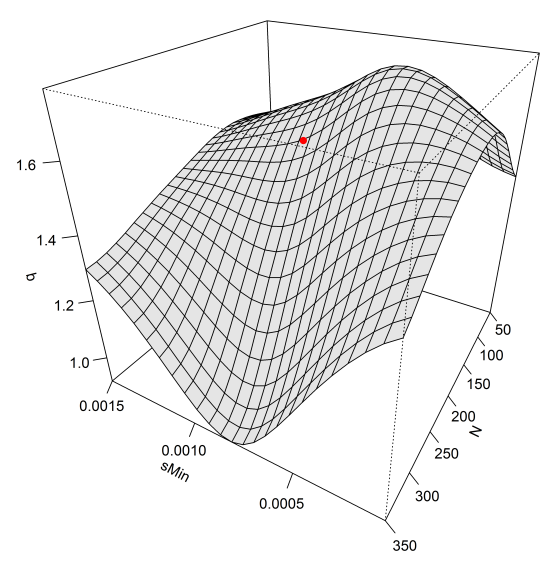

(b) $3 \mathrm{D}$ response surface (Laplace)

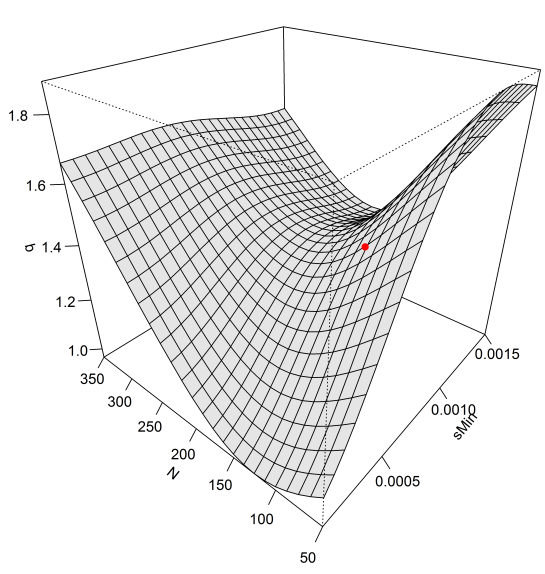

(e) 3D response surface (Gaussian)

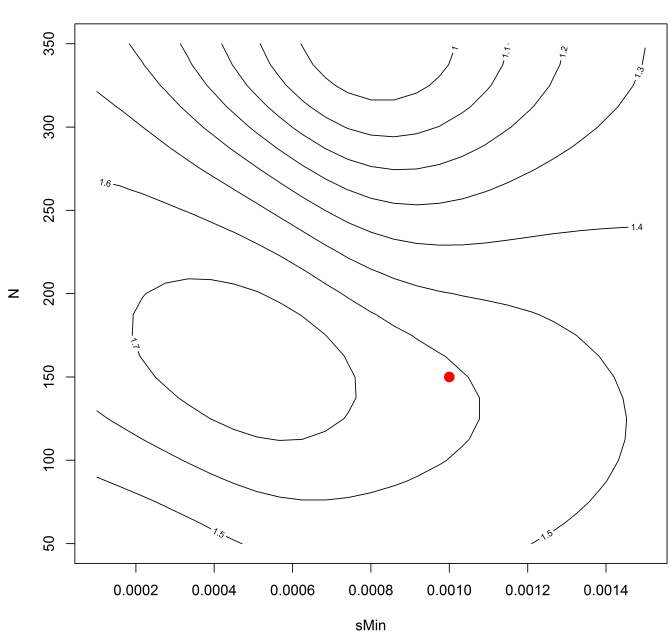

(c) Isolevels response surface (Laplace)

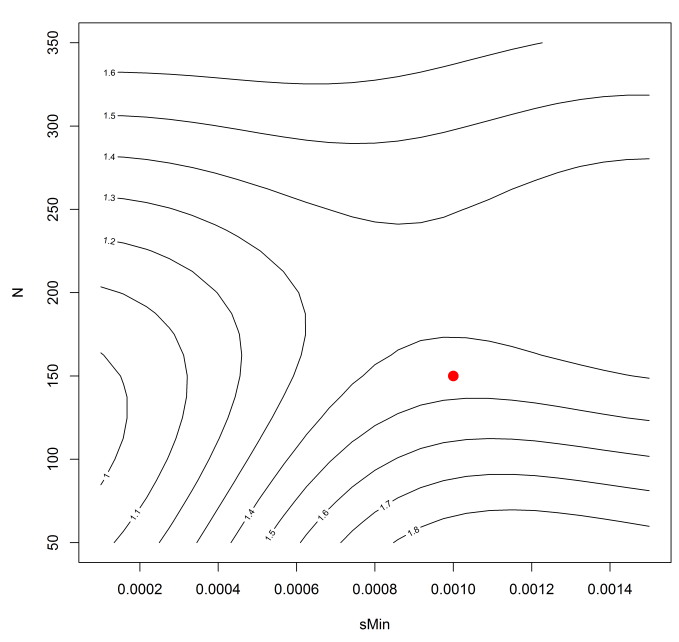

(f) Isolevels response surface (Gaussian)

Figure 5: Sensitivity analysis and response surfaces - Laplace and Gaussian shocks. All remaining parameters set at default settings (round mark at default $s_{\min }, N$ and $\mu$ ). 


\section{Discussion}

Our results show that the model is able to reproduce, over most of the parameters space, fat-tailed growth rates distributions - and even strict Laplace ones. The Kriging meta-models confirm and strengthen the results obtained in Dosi et al., (2015), providing evidence that the coupling of the two evolutionary processes of learning and selection is a strong candidate to explain the observed fat-tailed distributions of firm growth rates.

From the analysis made possible by the meta-models, the modeller can acquire a set of relevant new information on the original model behaviour. However, care should be taken to account for the expected prediction errors on the response surfaces: isolevels and 3D surfaces should be understood with the associated confidence intervals (at the desired significance level), that are not regular (constant) in Kriging meta-models. In any case, the order of magnitude of the out-of-sample RMSE in Table 4 remains a good indication of the limits to be expected on the overall confidence intervals. Moreover, even when the confidence intervals may be not sufficiently narrow to objectively accept or reject a given proposition, the topological information provided by the meta-model response surface has proved to be a powerful tool on guiding (and making possible) the exploration of the original model by means of other (more data-demanding) tools, like conventional Monte Carlo sampling.

According to the global effect of parameters on meta-models responses, provided by variance decomposition, the elicited parameters in the three analysed cases in order of significance are: [i] $s_{\min }$ (exit market share), [ii] $A$ (replicator sensitivity), [iii] $N$ (number of firms), and [iv] $\gamma$ (degree of cumulativity). When they are relevant, according to the shocks distribution case, both direct and interaction effects influence the response surfaces. From the analysis of the latter, some regular patterns of the parameters' effects on the value of meta-models' $\hat{b}$ can be identified.

First, the $s_{\min }$ parameter exerts a mostly monotonic influence on the change of $\hat{b}$ : the higher the death-threshold the fatter the tails of growth rate distribution are. This result, admittedly unexpected in its strength, is likely to capture the impact of that extreme form of selection which is "death", upon the whole distribution of growth rates.

Second, the higher the value of the $A$ parameter, in general the lower the value of $\hat{b}$. Similarly to $s_{\text {min }}$, this parameter controls for the degree of selection operating among incumbent firms. In fact, higher selection in the market induces a greater reallocation of shares among surviving incumbents. In the region where competition is fierce both in the entry-exit and in the reallocation processes, characterised by high values for $s_{\text {min }}$ and $A$ respectively, very low levels of the $\hat{b}$ parameter are recorded and almost "pure" Laplacian tails emerge. 
Third, the mechanism of cumulation in learning activities, modulated by $\gamma$, exerts a positive influence on the tail-fatness in our meta-model specifications, as already detected in Dosi et al., (2015). The process of cumulation of knowledge influences directly firms productivity growth, and indirectly their performance in the market.

The results from the Kriging meta-models confirm and strengthen the previous findings discussed in Dosi et al., (2015) but a word of caution is necessary when interpreting the meta-model and in particular the effect of the coefficients $\psi_{g}$ on $\operatorname{corr}\left(y\left(\mathbf{x}_{i}\right), y\left(\mathbf{x}_{j}\right)\right)$. In fact, a simplification of the deterministic component $\lambda(\mathbf{x})$ puts the burden of explanation on the stochastic part $\delta(\mathbf{x})$. Admittedly, focusing on the modeling of $\lambda$ by, say, a traditional fixed effects polynomial regression would yield an increasing dimensionality of the meta-model, comprising all the $k=10,7$ or 6 parameters themselves, their interaction and higher order terms. Intuitively, the Kriging rationale in privileging the modeling of $\operatorname{cov}(\delta(\mathbf{x}))$, instead, is that it allows for the capture the behavior of $Y(\mathbf{x})$ using much fewer observations while still keeping global covariance-based sensitivity analysis possible. Indeed, a constant deterministic function can be not only the result of the sum of constant $x_{1}, \ldots, x_{k} \in$ $\mathbb{R}$ parameters but also, being $\lambda(\mathbf{x}): \mathbb{R}^{k} \rightarrow \mathbb{R}$ the function representing the global trend of the meta-model $Y$, it may well proximately capture different dynamics for some parameters $x_{g}$ : in such a case a constant deterministic component could "artificially" flatten the meta-model. Of course the associated loss of information about the model sharply falls as the number of parameters or of the DoE samples increase.

Furthermore, even if the correlation function coefficients are estimated using data coming from the original model, the ensuing covariances are fully precise only at the exact DoE (sampling) points, as for all others we are using an interpolation of the closest DoE points to predict the correlation values. That is why, in fact, the metamodel is just a surrogate model, an approximation which cannot - and so should not be used to - substitute the original model: the estimated coefficients in Table 4 represent the overall expected effects of each parameter $x_{g}$ on the variance of metamodel's response and thus in the final predicted values $Y(\mathbf{x})$, all subject to the usual restrictions of any non-parametric Bayesian approximation, in particular the chosen priors (the trend and the correlation functional forms). The coefficients $\psi$ being estimated govern "associations" among the original parameters (the covariation in the components of the random effect $\delta(\mathbf{x})$ ), but they do not represent directly (fixed) effects of the original parameters $x_{k}$ on $Y(\mathbf{x})$.

Notwithstanding these caveats, the meta-model approximate response surface is still a powerful guide for the general exploration of the original model, as a kind of "reduced map", providing illuminating guidance on the sign of the effects of the 
parameters on the output variable(s), on their relative importance, and on the ones critical for particular "suspicious" points. Relatedly, the exercise hints to the region of the parameters space to intensively search, on the ground of the original model, performing traditional local sensitivity analysis, at this stage more feasible given the lower number of dimensions and factor ranges. That is, despite some possible - or even likely - "false-positives" from the meta-models, any search in the original model becomes at least better informed with them.

\section{Conclusions}

Empirically, one ubiquitously observes fat-tailed distributions of firm growth rates. In Dosi et al., (2015) we built a simple multi-firm agent-based model able to reproduce this stylised fact. In this contribution we use Kriging meta-modeling methodology associated with a computationally efficient Near-Orthogonal Latin Hypercube design of experiment which allows for the fully simultaneous analysis of all of the models parameters under their entire useful ranges of variation. The exercise confirms the high level of generality of the results previously obtained by means of a statistically robust global sensitivity analysis. The mechanisms of market selection, both in the entry-exit and in the market share reallocation processes, together with cumulative learning, turn out to be quite robust candidates to explain the tent-shaped distribution of firms' growth rates.

\section{Acknowledgements}

We thank Francesca Chiaromonte for helpful comments and discussions. We gratefully acknowledge the support by the European Union's Horizon 2020 research and innovation programme under grant agreement No. 649186 - ISIGrowth and by Fundação de Amparo à Pesquisa do Estado de São Paulo (FAPESP), process No. 2015/09760-3.

\section{References}

Bargigli, L., L. Riccetti, A. Russo, and M. Gallegati (2016). Network Calibration and Metamodeling of a Financial Accelerator Agent Based Model. Available at SSRN.

Bottazzi, G., E. Cefis, and G. Dosi (2002). "Corporate growth and industrial structures: some evidence from the Italian manufacturing industry". Industrial and Corporate Change 11.4, 705-723.

Bottazzi, G. and A. Secchi (2003). "A stochastic model of firm growth". Physica A: Statistical Mechanics and its Applications 324.1, 213-219. 
Bottazzi, G. and A. Secchi (2006). "Explaining the distribution of firm growth rates". RAND Journal of Economic 37.2, 235-256.

Bottazzi, Giulio (2014). SUBBOTOOLS. Scuola Superiore Sant'Anna. Pisa, Italy. URL: http://cafim.sssup.it/〜giulio/software/subbotools/.

Cioppa, T. and T. Lucas (2007). "Efficient nearly orthogonal and space-filling Latin hypercubes". Technometrics 49.1, 45-55.

Dosi, G. (2007). "Statistical Regularities in the Evolution of industries. A guide trough some evidence and challenges for the theory". In: Perspectives on innovation. Malerba, F. and Brusoni, S. Editors (2007).

Dosi, G., O. Marsili, L. Orsenigo, and R. Salvatore (1995). "Learning, market selection and the evolution of industrial structures". Small Business Economics 7.6, 411-436.

Dosi, G., R. Nelson, and S. Winter (2000). The nature and dynamics of organizational capabilities. Oxford University Press.

Dosi, G., M.C. Pereira, and M.E. Virgillito (2015). The footprint of evolutionary processes of learning and selection upon the statistical properties of industrial dynamics. LEM Papers Series 2015/04. Laboratory of Economics and Management (LEM), Sant'Anna School of Advanced Studies, Pisa, Italy.

Dupuy, D., C. Helbert, and J. Franco (2015). "DiceDesign and DiceEval: Two R Packages for Design and Analysis of Computer Experiments". Journal of Statistical Software 65.11, 1-38. URL: http://www . jstatsoft.org/v65/i11/.

Hanusch, H. and A. Pyka (2007). Elgar Companion to Neo-Schumpeterian Economics. Edward Elgar.

Helton, J., J. Johnson, C. Sallaberry, and C. Storlie (2006). "Survey of samplingbased methods for uncertainty and sensitivity analysis". Reliability Engineering E System Safety 91.10, 1175-1209.

Ijiri, Y. and H. Simon (1977). Skew Distributions and the Sizes of Business Firms. Amsterdam: North-Holland.

Iooss, B., L. Boussouf, V. Feuillard, and A. Marrel (2010). "Numerical studies of the metamodel fitting and validation processes". arXiv preprint arXiv:1001.1049.

Jeong, S., M. Murayama, and K. Yamamoto (2005). "Efficient optimization design method using kriging model". Journal of Aircraft 42.2, 413-420.

Kleijnen, J. and R. Sargent (2000). "A methodology for fitting and validating metamodels in simulation". European Journal of Operational Research 120.1, 14-29.

Krige, D. (1951). "A statistical approach to some basic mine valuation problems on the Witwatersrand". Journal of the Chemical, Metallurgical and Mining Society of South Africa 52.6, 119-139.

Malerba, F. and S. Brusoni (2007). Perspectives on innovation. Cambridge University Press. 
Matheron, G. (1963). "Principles of geostatistics". Economic geology 58.8, 12461266.

McKay, M., R. Beckman, and W. Conover (2000). "A comparison of three methods for selecting values of input variables in the analysis of output from a computer code". Technometrics 42.1, 55-61.

Metcalfe, J. S. (1998). Evolutionary Economics and Creative Destruction. Routledge.

Nelson, R. R. and S. G. Winter (1982). An Evolutionary Theory of Economic Change. Cambridge, MA: Belknap Press of Harvard University Press.

R Core Team (2016). R: A Language and Environment for Statistical Computing. R Foundation for Statistical Computing. Vienna, Austria. URL: https://www.Rproject.org/.

Rasmussen, C. and C. Williams (2006). Gaussian processes for machine learning. Cambridge, MA: MIT Press.

Roustant, O., D. Ginsbourger, and Y. Deville (2012). "DiceKriging, DiceOptim: Two $\mathrm{R}$ packages for the analysis of computer experiments by kriging-based metamodeling and optimization". Journal of Statistical Software 51.1, 1-55.

Salle, I. and Y. Murat (2014). "Efficient Sampling and Meta-Modeling for Computational Economic Models". Computational Economics 44.4, 507-536.

Saltelli, A. and P. Annoni (2010). "How to avoid a perfunctory sensitivity analysis". Environmental Modelling \& Software 25.12, 1508-1517.

Saltelli, A., M. Ratto, T. Andres, F. Campolongo, J. Cariboni, D. Gatelli, M. Saisana, and S. Tarantola (2008). Global sensitivity analysis: the primer. New York: John Wiley \& Sons.

Saltelli, A., S. Tarantola, and F. Campolongo (2000). "Sensitivity analysis as an ingredient of modeling". Statistical Science 15.4, 377-395.

Schumpeter, J. (1947). Capitalism, Socialism, and Democracy. New York and London: Harper \& Brothers Publishers.

Silverberg, G., G. Dosi, and L. Orsenigo (1988). "Innovation, Diversity and Diffusion: A Self-organisation Model". Economic Journal 98.393, 1032-54.

Valente, Marco (2014). LSD: Laboratory for Simulation Development. University of L'Aquila. L'Aquila, Italy. URL: https://www.labsimdev.org/.

Van Beers, W. and J. Kleijnen (2004). "Kriging interpolation in simulation: a survey". In: Simulation Conference, 2004. Proceedings of the 2004 Winter. Vol. 1. IEEE.

Wang, G. and S. Shan (2007). "Review of metamodeling techniques in support of engineering design optimization". Journal of Mechanical design 129.4, 370-380. 